\title{
Nonlinear Dynamic Model-Based Adaptive Control of a Solenoid-Valve System
}

\author{
DongBin Lee, Peiman Naseradinmousavi, and C. Nataraj \\ Department of Mechanical Engineering and Center for Nonlinear Dynamics \& Control (CENDAC), Villanova University, \\ Villanova, PA 19085, USA \\ Correspondence should be addressed to DongBin Lee, dongbin.lee@villanova.edu
}

Received 6 December 2011; Revised 8 March 2012; Accepted 30 March 2012

Academic Editor: Lili Ma

Copyright ( 92012 DongBin Lee et al. This is an open access article distributed under the Creative Commons Attribution License, which permits unrestricted use, distribution, and reproduction in any medium, provided the original work is properly cited.

In this paper, a nonlinear model-based adaptive control approach is proposed for a solenoid-valve system. The challenge is that solenoids and butterfly valves have uncertainties in multiple parameters in the nonlinear model; various kinds of physical appearance such as size and stroke, dynamic parameters including inertia, damping, and torque coefficients, and operational parameters especially, pipe diameters and flow velocities. These uncertainties are making the system not only difficult to adjust to the environment, but also further complicated to develop the appropriate control approach for meeting the system objectives. The main contribution of this research is the application of adaptive control theory and Lyapunov-type stability approach to design a controller for a dynamic model of the solenoid-valve system in the presence of those uncertainties. The control objectives such as set-point regulation, parameter compensation, and stability are supposed to be simultaneously accomplished. The error signals are first formulated based on the nonlinear dynamic models and then the control input is developed using the Lyapunov stability-type analysis to obtain the error bounded while overcoming the uncertainties. The parameter groups are updated by adaptation laws using a projection algorithm. Numerical simulation results are shown to demonstrate good performance of the proposed nonlinear modelbased adaptive approach and to compare the performance of the same solenoid-valve system with a non-adaptive method as well.

\section{Introduction}

In order to achieve advanced automation [1] in systems such as marine vessels or ship-based machinery system [2], solenoid actuators and valves are often used [3] to increase survivability and capability. One typical type of actuator driven by solenoids is shown in Figure 1, which is operated by the electromagnetic force. The electric-driven solenoid valve system [4] and its sophisticated control can provide high levels of automation in large systems. The useful function of the solenoid-valve, once an electrical signal (current or voltage) is applied, is to activate a mechanical motion such as displacement or rotation via the solenoid magnetic forces and torques. The proportional solenoids normally require integrated electronics for controlling the plunger to give such a signal. Hydrodynamic torque of a butterfly valve comprises the core knowledge of fluid valve system design [5], and it is known that most of the valves in real systems have strongly nonlinear characteristics between the force and displacement $[6,7]$. The use of an intelligent approach $[8,9]$ such as adaptive, robust, optimal, or nonlinear control of the actuator-valve machinery systems will benefit a wide spectrum of nonlinear systems, compensating for nonlinearities [10] and dynamic characteristics. This approach will not only decrease the amount of cost and casualties but also improve the performance of the mechatronic system. To investigate the particular application, it is important to emphasize the nonlinear dynamic modeling analysis of such actuatorvalve systems because the accuracy and reliability of these systems depend highly on the mathematical system modeling [11] and its validation. In [12], the authors developed and analyzed the nonlinear dynamic model of a solenoid-valve system; the reader is also referred to $[13,14]$ for recent modeling and analysis of solenoid actuators.

This paper will focus on model-based nonlinear adaptive control of an actuator-butterfly valve. The solenoid-valve system is described based on the exact model knowledge of the system. Figure 1 shows the integrated system, which 


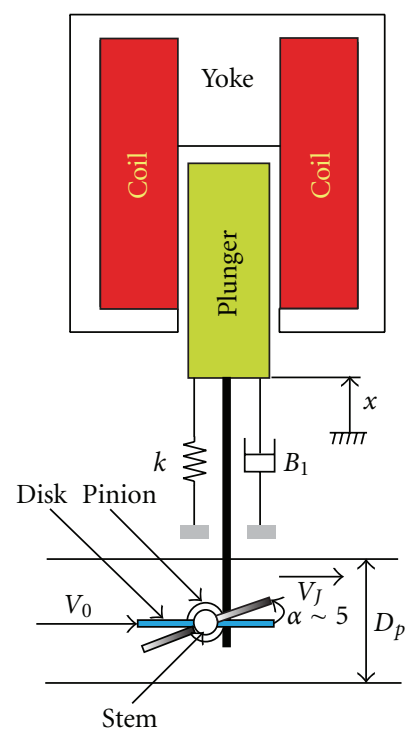

FIGURE 1: System Configuration.

consists of an electric-driven solenoid and a butterfly valve. The valve operates by solenoids that use a magnetic coil to move a movable plunger connected with the valve stem by means of a gear train and linkage. The control input is designed by substituting the current signal from the model of the electromagnetic force, pulling the plunger, and then controlling the angular position of the butterfly valve. The system has uncertainties in multiple parameters in the dynamic model, which requires the system to continuously adjust to the environment and consequently requires adaptation for sustainability and capability. The integrated system is highly nonlinear in addition to its parameter uncertainties. Hence, an adaptation law is proposed $[8,9,17]$ and an adaptive control method is developed for the solenoid-valve system in multiple parametric uncertainties. A closed-loop stable controller is designed for the set-point trajectory tracking by introducing a Lyapunov-based stability analysis [9] based on the error signals of the nonlinear solenoid-valve system. The numerical results in the simulation are used for initial verification and performance evaluation.

\section{Model-Based Nonlinear System}

2.1. System Model. The dynamic equations of motion of the plunger and butterfly valve are given by [15]

$$
\begin{gathered}
m \ddot{x}+B_{1} \dot{x}+k x=F_{\mathrm{mag}}-F_{c}, \\
J \ddot{a}+B_{2} \dot{\alpha}=r_{g} F_{c}-T_{\mathrm{tot}},
\end{gathered}
$$

where $x(t)$ is the displacement of the solenoid plunger, $\alpha(t)$ is the angle of butterfly disk, and $T_{\text {tot }}(t)$ is the sum of the hydrodynamic and bearing torques expressed as $T_{\text {tot }}=T_{b}+T_{h}$. The bearing torque is given as $T_{b}=(\pi / 8) \mu D_{s} D_{p}^{2} \Delta P_{\nu} C_{R}(\alpha)$, where the bearing toque coefficient, $C_{R}(\alpha)=\sqrt{C_{L}^{2}+C_{D}^{2}}=$ $\sqrt{\left(1.1 \sqrt[4]{\sin \left((\alpha / 90)^{3} 180\right)}\right)^{2}+(\sqrt[3]{\cos \alpha})^{2}}$, is obtained from the valve modeling (see $[5,15])$ and the two subterms, lifting force $C_{L}=1.1 \sqrt[4]{\sin \left((\alpha / 90)^{3} 180\right)}$ and drag force $C_{D}=\sqrt[3]{\cos \alpha}$, are nonlinear functions of the valve angle rotation $\alpha(t)$. The hydrodynamic torque $T_{h}$ is obtained by reviewing threedimensional hydrodynamic torque coefficient based on [7, 16] as $T_{h}=(8 / 3 \pi) \rho D_{p}^{3} V_{O}^{2} T_{c}(\alpha)\left[\left(V_{J} / V_{O}\right)(\alpha)\right]^{2}$, where both $T_{c}(\alpha)$ and $\left(V_{J} / V_{O}\right)(\alpha)$ depend on the closing angle $(\alpha)$ of the butterfly valve and $D_{p}^{3}$ term is a nonlinear term according to the pipe size. Solving the two equations in (1) with the contact force, $F_{c}(t)$, and substituting the magnetic force, $F_{\text {mag }}(t)$, into the equation yields

$$
\begin{gathered}
\left(m+\frac{J}{r_{g}^{2}}\right) r_{g} \ddot{x}+\left(B_{1}+\frac{B_{2}}{r_{g}^{2}}\right) r_{g} \dot{x}+k r_{g} x \\
=r_{g} \frac{C_{2} N^{2}}{2\left(C_{1}+C_{2} x\right)^{2}} i^{2}-T_{\text {tot }},
\end{gathered}
$$

where the magnetic force $F_{\mathrm{mag}}=\left(C_{2} N^{2} / 2\left(C_{1}+C_{2} x\right)^{2}\right) i^{2}$ used to lift the plunger of the solenoid actuator. The actuator is a current-controlled solenoid [15], proportional to the square of the current $i(t)$, and $C_{1}, C_{2}$ are reluctances of the magnetic paths, obtained from the geometry of electric actuator $[4,15]$. It is assumed that the pinion and the valve are moving at the same speed, that is, the gear ratio is $1: 1$ and $x(t)=r_{g} \alpha$ is the simple geometric relationship between the displacement of the pinion and the valve angle. Hence, the current source $i^{2}(t)$ is substituted for designing the closedloop control input, $u(t)$, and then the following equation is obtained:

$$
\begin{gathered}
\left(m+\frac{J}{r_{g}^{2}}\right) r_{g} \ddot{x}+\left(B_{1}+\frac{B_{2}}{r_{g}^{2}}\right) r_{g} \dot{x}+k r_{g} x \\
=r_{g} \frac{C_{2} N^{2}}{2\left(C_{1}+C_{2} x\right)^{2}} u-T_{\text {tot. }}
\end{gathered}
$$

For the subsequent controller design, multiplying (3) with the inverse term of the control input, $2\left(C_{1}+C_{2} x\right)^{2}$ / $\left(r_{g} C_{2} N^{2}\right)$, yields a compact form of the dynamic equation as

$$
M(x, \theta) \ddot{x}+C(x, \theta) \dot{x}+D(x, \theta) x=u-B\left(T_{b}+T_{h}\right),
$$

where $\theta$ describes a lumped expression of parameters obtained from (3) and (4), where each parameter is shown in Table 2 and the substituted terms are defined as follows:

$$
\begin{gathered}
M(x, \theta)=\left(m+\frac{J}{r_{g}^{2}}\right) r_{g} B, \quad B=\frac{2\left(C_{1}+C_{2} x\right)^{2}}{r_{g} C_{2} N^{2}}, \\
C(x, \theta)=\left(B_{1}+\frac{B_{2}}{r_{g}^{2}}\right) r_{g} B, \quad D(x, \theta)=k r_{g} B .
\end{gathered}
$$

2.2. Error Signals Formulation. The following set-point control approach is used. Let $x_{d}(t)$ define the set-point trajectory and then the error can be defined as

$$
e \equiv x_{d}-x, \quad \dot{e}=\dot{x}_{d}-\dot{x}, \quad \ddot{e}=\ddot{x}_{d}-\ddot{x},
$$




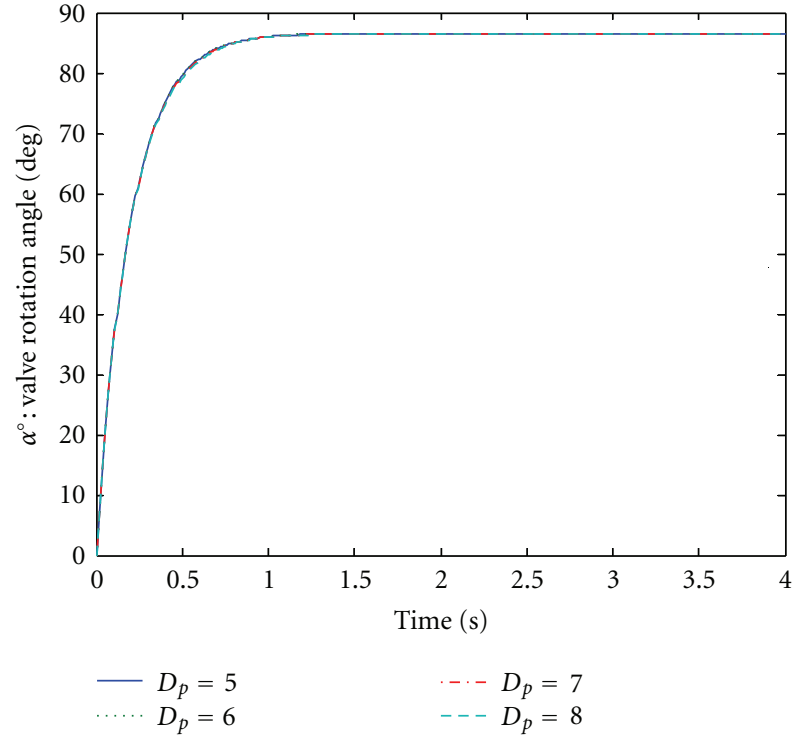

FIGURE 2: Valve rotation angle $\alpha(t)$ (adaptive approach).

where $\dot{x}_{d}(t)$ and $\ddot{x}_{d}(t)$ are the first and second time derivatives of $x_{d}(t)$, which are assumed to be bounded. Premultiplying $\ddot{e}(t)$ in the last error signal of (6) with $M(x, \theta)$ yields $M(x, \theta) \ddot{e}=M(x, \theta) \ddot{x}_{d}-M(x, \theta) \ddot{x}$. Substituting $M(x, \theta) \ddot{x}$ in (4) into the above equation produces

$$
\begin{aligned}
M(x, \theta) \ddot{e}= & M(x, \theta) \ddot{x}_{d}+C(x, \theta) \dot{x}+D(x, \theta) x-u \\
& +B\left(T_{b}+T_{h}\right) .
\end{aligned}
$$

A filtered error signal and its derivative are defined as

$$
r \equiv \dot{e}+\lambda_{1} e, \quad \dot{r}=\ddot{e}+\lambda_{1} \dot{e},
$$

where $\lambda_{1} \in \mathfrak{R}^{+}$is a positive adjustable control gain. Multiplying (8) with $M(x, \theta)$ and then substituting for $M(x, \theta) \ddot{e}(t)$ in (7) yields

$$
\begin{aligned}
M(x, \theta) \dot{r}= & M(x, \theta) \ddot{x}_{d}+C(x, \theta) \dot{x}+D(x, \theta) x-u \\
& +B\left(T_{b}+T_{h}\right)+M(x, \theta) \lambda_{1} \dot{e}+\frac{1}{r_{g}^{2}} e-\frac{1}{r_{g}^{2}} e,
\end{aligned}
$$

where the last term $e(t) / r_{g}^{2}$ is added and subtracted for further development of the control design based on Lyapunov's method.

\section{Lyapunov-Based Adaptive Feedback Control}

Let $V(t)$ be a Lyapunov candidate function

$$
V=\frac{1}{2}\left(r^{T} M r+e^{T} e+e_{\alpha}^{T} e_{\alpha}+\widetilde{\Theta}^{T} \Gamma^{-1} \widetilde{\Theta}\right),
$$

\begin{tabular}{|c|c|c|c|}
\hline No. & Terms & No. & Terms \\
\hline$W_{101}$ & $\ddot{x}_{d}$ & $W_{113}$ & $C_{R}(\alpha) x$ \\
\hline$W_{102}$ & $\ddot{x}_{d} x$ & $W_{114}$ & $T_{c}(\alpha)\left[V_{J}(\alpha) / V_{O}\right]^{2} x^{2}$ \\
\hline$W_{103}$ & $\ddot{x}_{d} x^{2}$ & $W_{115}$ & $C_{R}(\alpha) x^{2}$ \\
\hline$W_{104}$ & $\dot{x}$ & $W_{116}$ & $\dot{x} \dot{e}$ \\
\hline$W_{105}$ & $\dot{x} x$ & $W_{117}$ & $\dot{x} e$ \\
\hline$W_{106}$ & $\dot{x} x^{2}$ & $W_{118}$ & $x \dot{x} \dot{e}$ \\
\hline$W_{107}$ & $x$ & $W_{119}$ & $x \dot{x} e$ \\
\hline$W_{108}$ & $x^{2}$ & $W_{120}$ & $e$ \\
\hline$W_{109}$ & $x^{3}$ & $W_{121}$ & $\lambda_{1} \dot{e}$ \\
\hline$W_{110}$ & $T_{c}(\alpha)\left[V_{J}(\alpha) / V_{O}\right]^{2}$ & $W_{122}$ & $\lambda_{1} x \dot{e}$ \\
\hline$W_{111}$ & $C_{R}(\alpha)$ & $W_{123}$ & $\lambda_{1} x^{2} \dot{e}$ \\
\hline$W_{112}$ & $T_{c}(\alpha)\left[V_{J}(\alpha) / V_{O}\right]^{2} x$ & - & - \\
\hline$\widehat{\Theta}_{101}$ & $\widehat{M}_{s} 2 \hat{C}_{1}^{2} /\left(\widehat{C}_{2} \hat{N}^{2}\right)$ & $\widehat{\Theta}_{113}$ & $\widehat{T}_{b 1} 4 \widehat{C}_{1} /\left(\hat{r}_{g} \hat{N}^{2}\right)$ \\
\hline$\hat{\Theta}_{102}$ & $\widehat{M}_{s} 4 \widehat{C}_{1} / \widehat{N}^{2}$ & $\widehat{\Theta}_{114}$ & $\widehat{T}_{h 1} 2 \widehat{C}_{2} /\left(\hat{r}_{g} \hat{N}^{2}\right)$ \\
\hline$\widehat{\Theta}_{103}$ & $\widehat{M}_{s} 2 \widehat{C}_{2} / \widehat{N}^{2}$ & $\widehat{\Theta}_{115}$ & $\widehat{T}_{b 1} 2 \widehat{C}_{2} /\left(\hat{r}_{g} \hat{N}^{2}\right)$ \\
\hline$\widehat{\Theta}_{104}$ & $\widehat{C}_{s} 2 \widehat{C}_{1}^{2} /\left(\widehat{C}_{2} \widehat{N}^{2}\right)$ & $\widehat{\Theta}_{116}$ & $\widehat{M}_{s} 2 \widehat{C}_{1} / \widehat{N}^{2}$ \\
\hline$\widehat{\Theta}_{105}$ & $\hat{C}_{s} 4 \widehat{C}_{1} / \hat{N}^{2}$ & $\widehat{\Theta}_{117}$ & $\widehat{M}_{s} 2 \widehat{C}_{1} / \widehat{N}^{2}$ \\
\hline$\hat{\Theta}_{106}$ & $\hat{C}_{s} 2 \hat{C}_{2} / \widehat{N}^{2}$ & $\widehat{\Theta}_{118}$ & $\widehat{M}_{s} 2 \widehat{C}_{2} / \widehat{N}^{2}$ \\
\hline$\widehat{\Theta}_{107}$ & $\hat{k} 2 \hat{C}_{1}^{2} /\left(\hat{C}_{2} \hat{N}^{2}\right)$ & $\widehat{\Theta}_{119}$ & $\widehat{M}_{s} 2 \widehat{C}_{2} / \widehat{N}^{2}$ \\
\hline$\widehat{\Theta}_{108}$ & $\hat{k} 4 \widehat{C}_{1} / \widehat{N}^{2}$ & $\widehat{\Theta}_{120}$ & $1 / r_{g}^{2}$ \\
\hline$\widehat{\Theta}_{109}$ & $\hat{k} 2 \widehat{C}_{2} / \widehat{N}^{2}$ & $\widehat{\Theta}_{121}$ & $\widehat{M}_{s} 2 \widehat{C}_{1}^{2} /\left(\widehat{C}_{2} \widehat{N}^{2}\right)$ \\
\hline$\widehat{\Theta}_{110}$ & $\widehat{T}_{h 1} 2 \hat{C}_{1}^{2} /\left(\hat{r}_{g} \widehat{C}_{2} \hat{N}^{2}\right)$ & $\widehat{\Theta}_{122}$ & $\widehat{M}_{s} 4 \widehat{C}_{1} / \widehat{N}^{2}$ \\
\hline$\widehat{\Theta}_{111}$ & $\widehat{T}_{b 1} 2 \hat{C}_{1}^{2} /\left(\hat{r}_{g} \hat{C}_{2} \hat{N}^{2}\right)$ & $\widehat{\Theta}_{123}$ & $\widehat{M}_{s} 2 \widehat{C}_{2} / \widehat{N}^{2}$ \\
\hline$\widehat{\Theta}_{112}$ & $\widehat{T}_{h 1} 4 \widehat{C}_{1} /\left(\hat{r}_{g} \hat{N}^{2}\right)$ & - & - \\
\hline
\end{tabular}

where the last term of the Lyapunov candidate function, $\Gamma=$ $\gamma I_{p \times p}$, is a constant diagonal matrix with the gain value $\gamma$, $I_{p \times p}$ is a $p \times p$ identity matrix, and the parameter estimation error, $\widetilde{\Theta}$, is defined as $\widetilde{\Theta}=\Theta-\widehat{\Theta}$, where $\Theta \in \mathfrak{R}^{p}$ is a known
TABLE 1: Regression and parameter estimation terms.

TABLE 2: List of simulation parameters.

\begin{tabular}{lccccccc}
\hline$m$ & $J$ & $r_{g}$ & $B_{1}$ & $B_{2}$ & $k$ & $N$ & $\Delta P_{v}$ \\
0.1 & $1.04 e^{-6}$ & $1 e^{-2}$ & 10 & 20 & $4 e^{2}$ & $8.8 e^{3}$ & 0.5 \\
\hline$C_{1}$ & $C_{2}$ & $\rho$ & $D_{p}$ & $V_{O}$ & $\mu$ & $D_{s}$ & $p$ \\
$1.57 e^{6}$ & $6.32 e^{8}$ & $1 e^{3}$ & $5^{\prime \prime} \sim 8^{\prime \prime}$ & 3.7 & 0.1 & 0.1 & 23 \\
\hline
\end{tabular}

constant parameter vector and $\widehat{\Theta} \in \mathfrak{R}^{p}$ is the estimated constant parameter vector (see Table 1). Differentiating (10) yields

$$
\dot{V}=r^{T} M \dot{r}+\frac{1}{2} r^{T} \dot{M} r+e^{T} \dot{e}+\frac{1}{r_{g}^{2}} e^{T} \dot{e}-\widetilde{\Theta}^{T} \Gamma^{-1} \dot{\hat{\Theta}},
$$

where the time derivative of the inertia matrix is obtained as $\dot{M}=\left(m+J / r_{g}^{2}\right)\left(4\left(C_{1}+C_{2} x\right) / N^{2}\right) \dot{x}$, where $\dot{B}=\left(4\left(C_{1}+\right.\right.$ $\left.\left.C_{2} x\right) / r_{g} N^{2}\right) \dot{x}, e_{\alpha}^{T} \dot{e}_{\alpha}=\left(1 / r_{g}^{2}\right) e^{T} \dot{e}$ as $e_{\alpha} \equiv \alpha_{d}-\alpha=x_{d} / r_{g}-$ $x / r_{g}=e / r_{g}, \dot{e}_{\alpha} \equiv \dot{\alpha}_{d}-\dot{\alpha}=\dot{x}_{d} / r_{g}-\dot{x} / r_{g}=\dot{e} / r_{g}$, in which the error signals of the valve angle, $e_{\alpha}(t)$, can be defined using the geometric relationship and $\dot{\widetilde{\Theta}}(t)=-\dot{\hat{\Theta}}$ comes from the definition of $\widetilde{\Theta}$. 


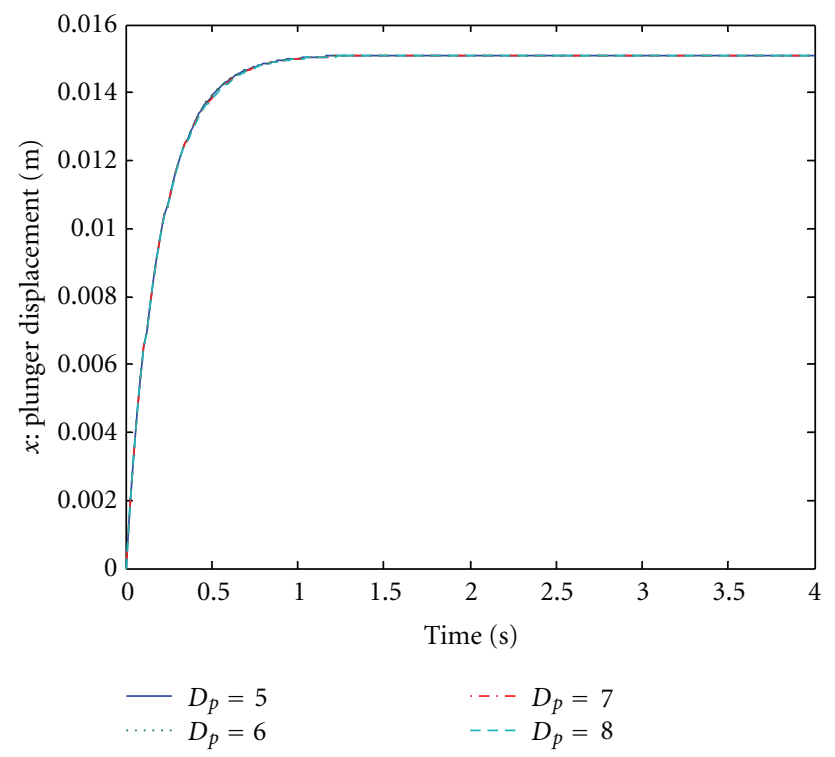

Figure 3: Plunger displacement $x(t)$ (adaptive approach).

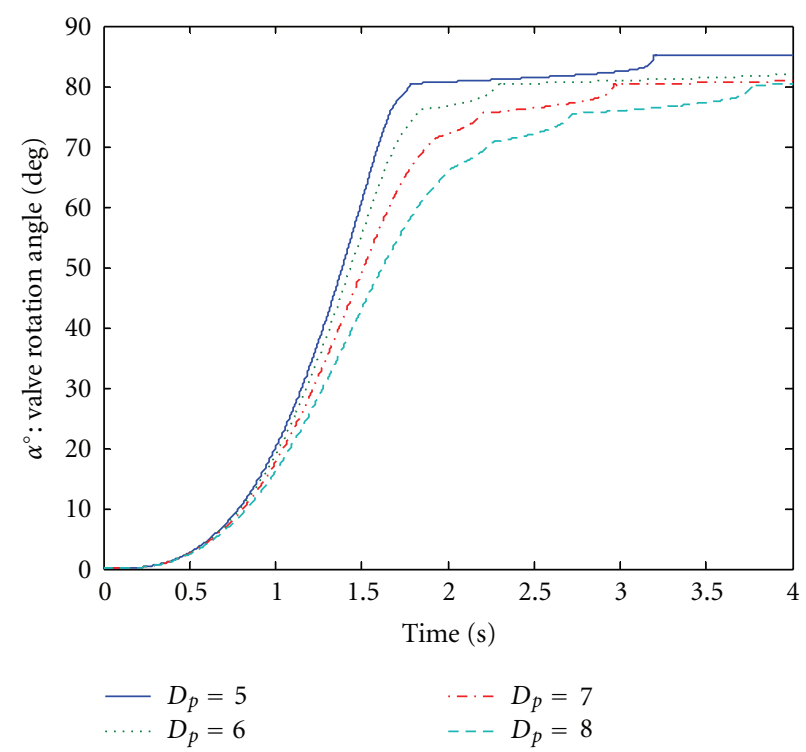

FIGURE 4: Valve rotation angle $\alpha(t)$ (no adaptation scheme).

3.1. Design of Control Input. Substituting $M(x, \theta) \dot{r}(t)$ into (11) yields

$$
\begin{aligned}
\dot{V}= & r^{T}\left(M \ddot{x}_{d}+C \dot{x}+D x-u+B\left(T_{b}+T_{h}\right)+M \lambda_{1} \dot{e}+\frac{1}{r_{g}^{2}} e\right) \\
& -\frac{(\dot{e}+e)^{T} e}{r_{g}^{2}}+\frac{1}{2} r^{T} \dot{M} r+e^{T} \dot{e}+\frac{1}{r_{g}^{2}} e^{T} \dot{e}-\widetilde{\Theta}^{T} \Gamma^{-1} \dot{\hat{\Theta}}
\end{aligned}
$$

where the last term in (9) premultiplied by $r(t)$ came out of the parenthesis in (12) and is used for the definition of $r(t)$

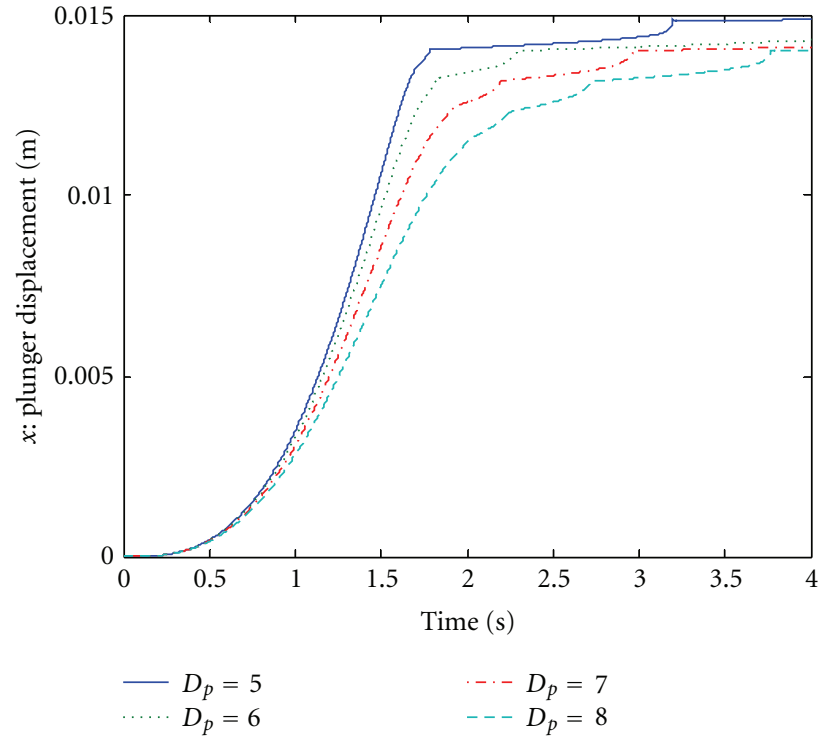

FIgURE 5: Plunger displacement $x(t)$ (no adaptation scheme).

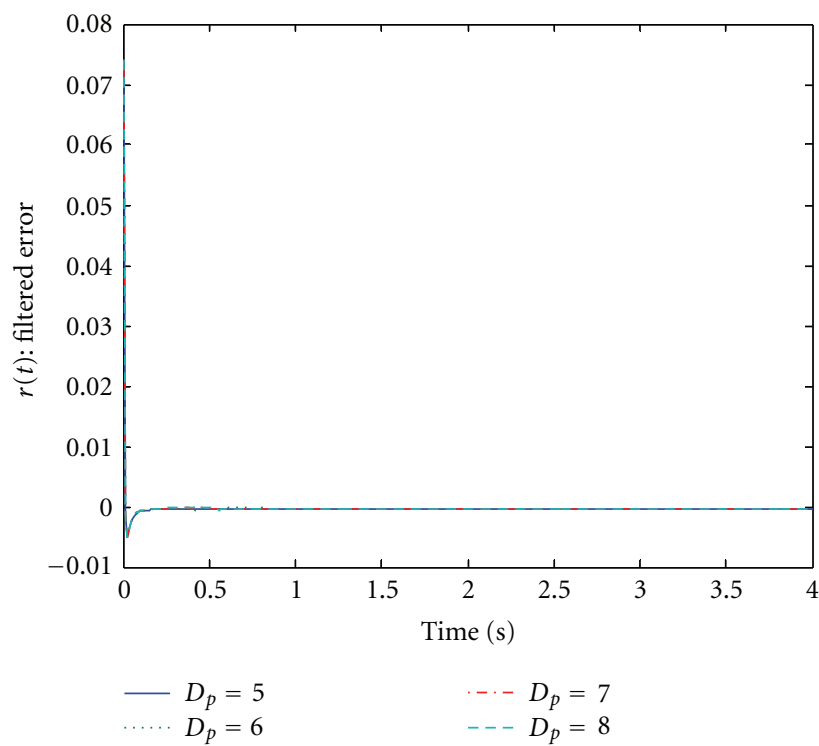

FiguRE 6: Filtered Error $r(t)$ (adaptive approach).

in (8). Then, combining the parameterized terms in (12) and substituting them into $W \Theta$ yields

$$
W \Theta=M \ddot{x}_{d}+C \dot{x}+D x+B\left(T_{b}+T_{h}\right)+M \lambda_{1} \dot{e}+\frac{1}{r_{g}^{2}} e+\frac{1}{2} \dot{M} r
$$

where $W\left(\ddot{x}_{d}, \dot{x}, x, r, \alpha, \dot{e}, e\right) \in \mathfrak{R}^{1 \times p}$ is a known regression vector, which is shown in the left side of Table 1 via the process given later (see (17)) and $\Theta$ as the nominal value of the lumped parameter vector. Rearranging (12) produces

$$
\dot{V}=r^{T}(W \Theta-u)-\frac{e^{T} e}{r_{g}^{2}}+e^{T} \dot{e}-\widetilde{\Theta}^{T} \Gamma^{-1} \dot{\hat{\Theta}},
$$




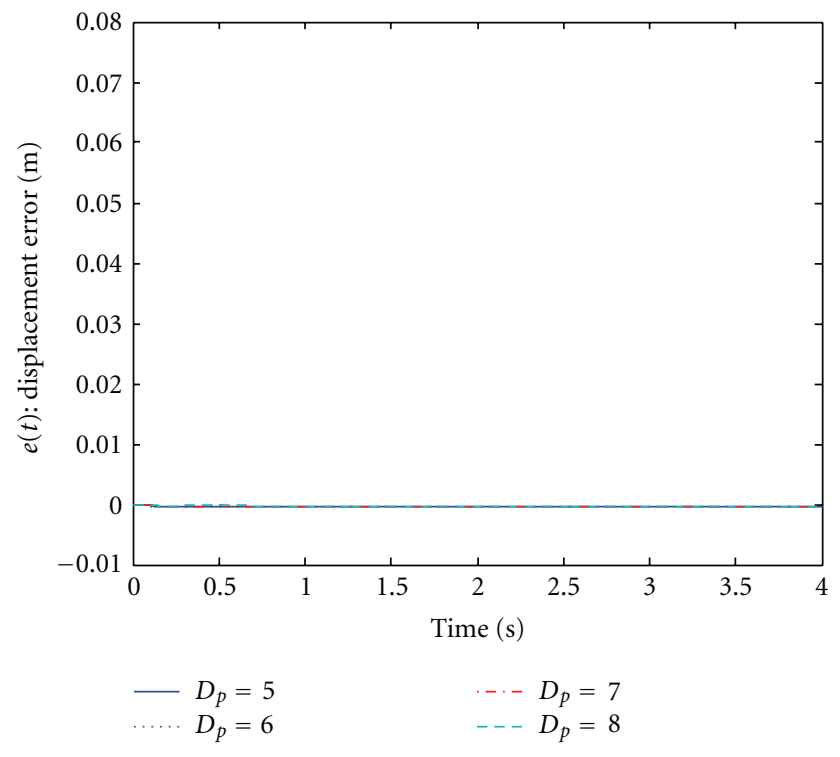

FIGURE 7: Displacement tracking error $e(t)$ (adaptive approach).

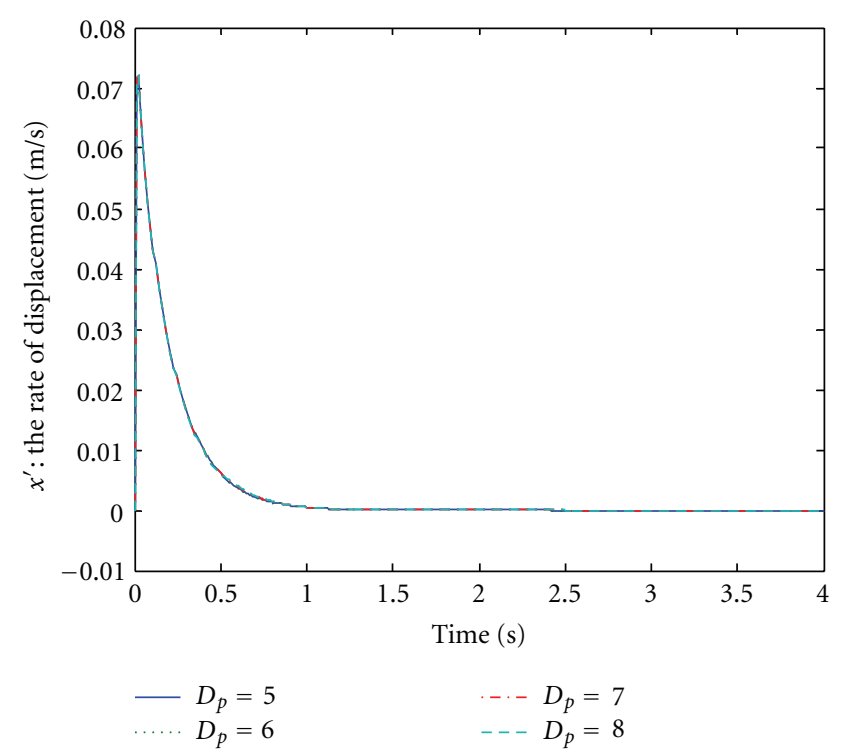

Figure 8: The rate of displacement $\dot{x}(t)$.

where $\dot{e}^{T} e / r_{g}^{2}$ is canceled in the last second term in (12), having the opposite sign because they are scalar, $\dot{e}^{T} e=e^{T} \dot{e}$.

The control input can be designed based on Lyapunov stability analysis, making $\dot{V}$ negative definite to be shown in the end, as

$$
u=W \widehat{\Theta}+k_{1} r+e,
$$

where $r(t)$ is a feedback error term, $k_{1}$ is a positive constant as the control gain, $e(t)$ is another feedback error term added to cancel the term having the opposite sign, $e^{T} \dot{e}$, outside the parenthesis by utilizing the definition of $r(t)$ given in (8), and $W \widehat{\Theta}$ captures the uncertainties associated with the elements

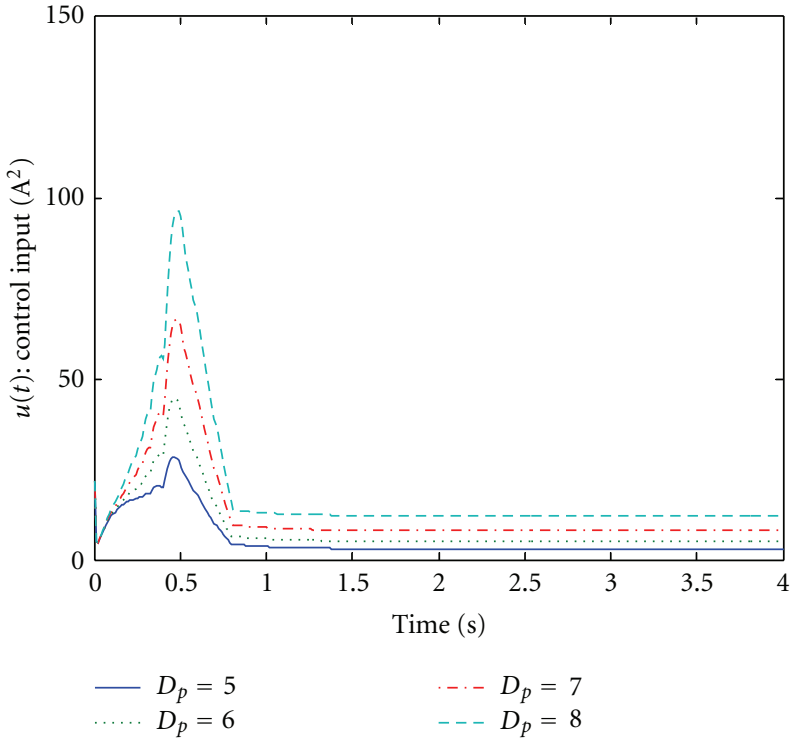

FIGURE 9: Control input $u(t)$ equivalent to square current $i^{2}(t)\left[\mathrm{A}^{2}\right]$.

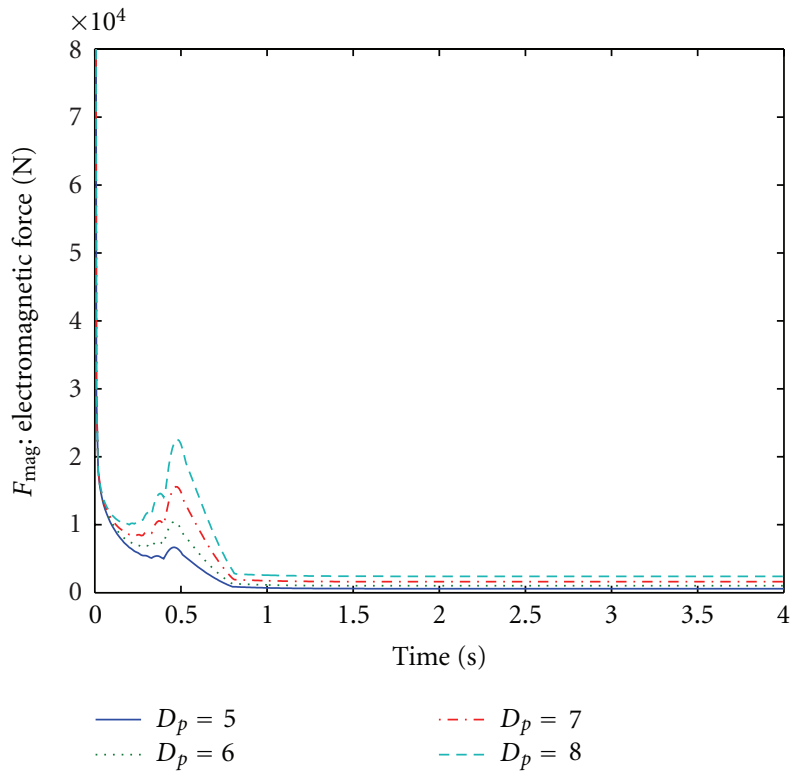

Figure 10: Electromagnetic force $F_{\text {mag }}(t)$.

of $M, C, D, B\left(T_{b 1}+T_{h 1}\right), M \lambda_{1}, 1 / r_{g}^{2}$, and $\dot{M}$, which is defined as

$$
\begin{aligned}
W \widehat{\Theta}= & \widehat{M} \ddot{x}_{d}+\hat{C} \dot{x}+\hat{D} x+\widehat{B}\left(\widehat{T}_{b 1} C_{R}(\alpha)+\widehat{T}_{h 1} T_{c}(\alpha)\left[\frac{V_{J}}{V_{O}}(\alpha)\right]^{2}\right) \\
& +\widehat{M} \lambda_{1} \dot{e}+\frac{1}{\hat{r}_{g}^{2}} e+\frac{1}{2} \dot{\widehat{M}} r
\end{aligned}
$$

where the estimated parameter sets are given as $\widehat{M}(x, \theta)=$ $\left(\hat{m}+\hat{J} / \hat{r}_{g}^{2}\right) \hat{r}_{g} \hat{B}$, where $\hat{B}=2\left(\widehat{C}_{1}+\widehat{C}_{2} x\right)^{2} / \widehat{r}_{g} \hat{C}_{2} \hat{N}^{2}, \hat{C}(x, \theta)=$ $\left(\widehat{B}_{1}+\widehat{B}_{2} / \widehat{r}_{g}^{2}\right) \widehat{r}_{g} \hat{B}, \hat{D}(x, \theta)=\hat{k} \hat{r}_{g} \widehat{B}, \widehat{T}_{b 1}=(\pi / 8) \hat{\mu} \widehat{D}_{s} \widehat{D}_{p}^{2} \Delta \hat{P}_{v}$, 


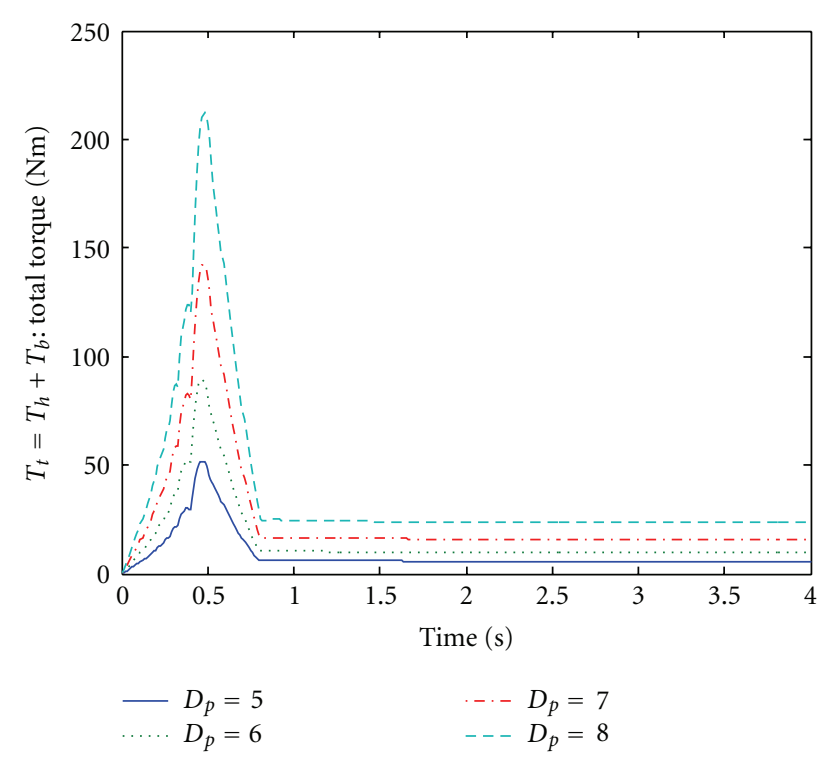

Figure 11: Total torque $T_{t}(t)$.

and $\widehat{T}_{h 1}=(8 / 3 \pi) \hat{\rho} \widehat{D}_{p}^{3} \hat{V}_{O}^{2}$. In order to develop the estimate parameter vector $\hat{\Theta}$ in (16), we need to first define the regression and the estimate terms. Thus, the first term, $\widehat{M} \ddot{x}_{d}$, in (16) can be defined as

$$
\begin{aligned}
& \widehat{M} \ddot{x}_{d}=\left(\hat{m}+\frac{\hat{J}}{\hat{r}_{g}^{2}}\right) \hat{r}_{g} \frac{2\left(\hat{C}_{1}+\hat{C}_{2} x\right)^{2}}{\hat{r}_{g} \hat{C}_{2} \hat{N}^{2}} \ddot{x}_{d} \\
& =\widehat{M}_{s} \frac{2 \widehat{C}_{1}^{2}}{\widehat{C}_{2} \hat{N}^{2}} \ddot{x}_{d}+\widehat{M}_{s} \frac{4 \widehat{C}_{1}}{\hat{N}^{2}} x \ddot{x}_{d}+\widehat{M}_{s} \frac{2 \widehat{C}_{2}}{\hat{N}^{2}} x^{2} \ddot{x}_{d} \\
& =\underbrace{\left[\begin{array}{lll}
\ddot{x}_{d} & x \ddot{x}_{d} & x^{2} \ddot{x}_{d}
\end{array}\right]}_{W_{101} \sim W_{103}} \underbrace{\left[\begin{array}{c}
\widehat{M}_{s} \frac{2 \widehat{C}_{1}^{2}}{\widehat{C}_{2} \hat{N}^{2}} \\
\widehat{M}_{s} \frac{4 \widehat{C}_{1}}{\hat{N}^{2}} \\
\widehat{M}_{s} \frac{2 \widehat{C}_{2}}{\hat{N}^{2}}
\end{array}\right]}_{\widehat{\Theta}_{101} \sim \hat{\Theta}_{103}},
\end{aligned}
$$

where $\widehat{M}_{s}=\left(\hat{m}+\hat{J} / \hat{r}_{g}^{2}\right), \widehat{C}_{s}=\left(\widehat{B}_{1}+\widehat{B}_{2} / \widehat{r}_{g}^{2}\right)$ (this is shown in Table 1), and $\hat{r}_{g}, \hat{C}_{1}$, or $\hat{C}_{2}$ are canceled. $W_{101} \sim W_{103}$ are the measurable regression terms and $\widehat{\Theta}_{101} \sim \widehat{\Theta}_{103}$ is the estimated parameters, defined in Table 1 , respectively. Similarly to (17), the rest of the terms in (16) are also given in Table 1.

3.2. Online Adaptation Laws for Parameter Updates. The following is constructed to define the known upper and lower bounds but with an unknown parameter of $\widehat{\Theta}(t)$ in the sense that

$$
\widehat{\Theta}_{-j} \leq \widehat{\Theta}_{j}(t) \leq \overline{\widehat{\Theta}}_{j}
$$

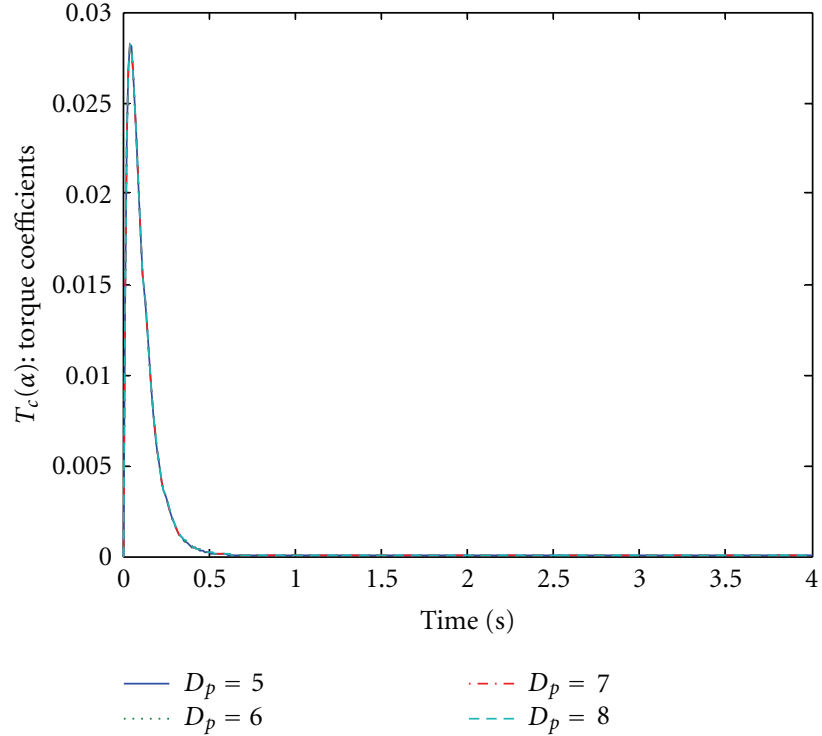

FIGURE 12: Hydrodynamic torque coefficient $T_{c}(\alpha)$.

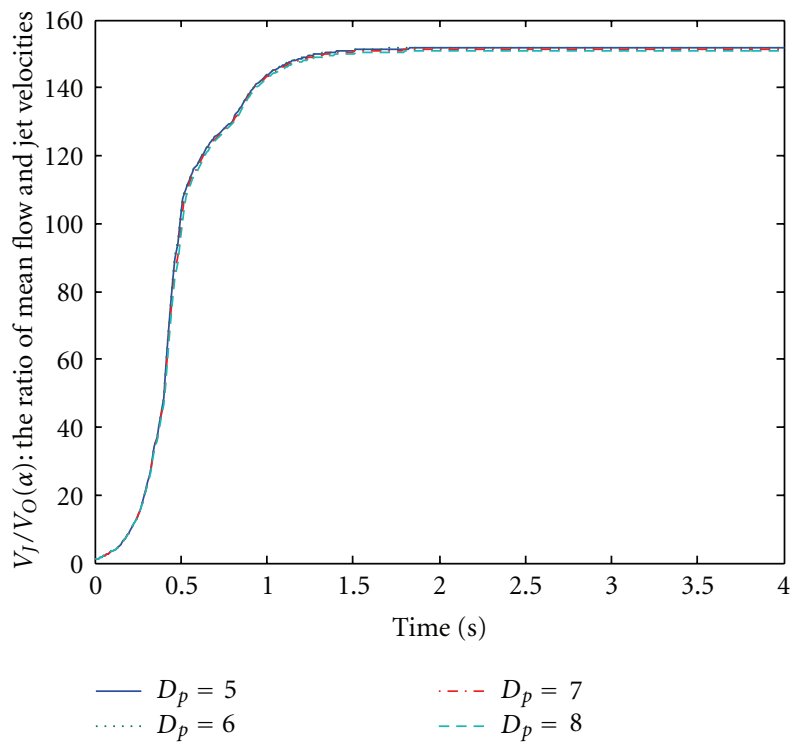

FIgure 13: The ratio of $V_{J}$ and $V_{O}:\left(V_{J} / V_{O}\right)(\alpha)$.

where $\widehat{\Theta}_{j}(t)$ are the estimated parameters as shown in Table 1 and $\widehat{\Theta}_{-j}$ and $\widehat{\widehat{\Theta}}_{j}$ are the lower and upper bounds of the estimated parameters, respectively, which will be set to the amount of percentage of their true values. The vector $\dot{\hat{\Theta}}_{j}(t)$ is designed to update using a projection-based algorithm as

$$
\dot{\hat{\Theta}}_{j}=\operatorname{Proj}\left\{\Gamma W^{T} r, \widehat{\Theta}_{j}\right\},
$$



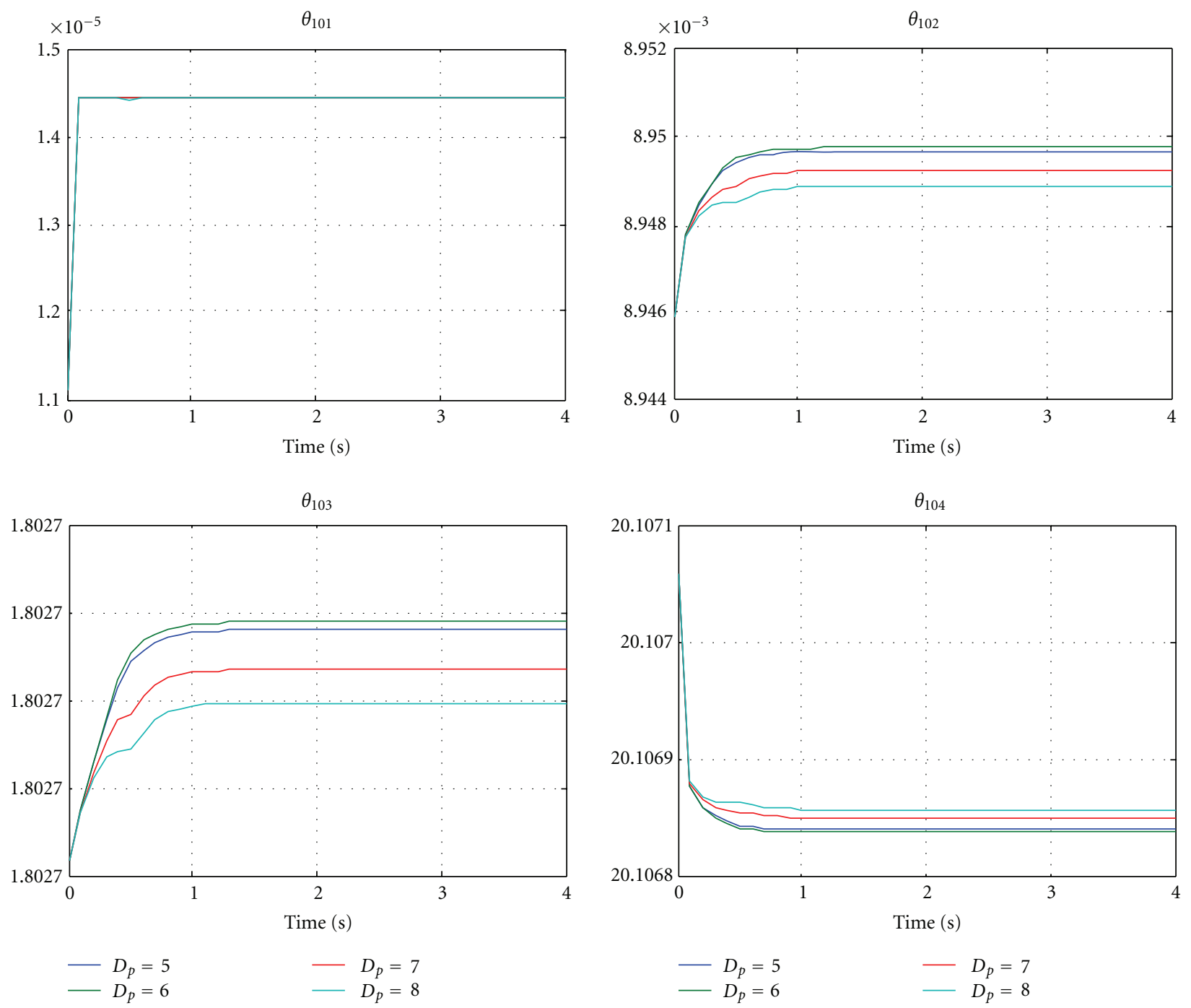

FIgURE 14: Parameter estimates: $\widehat{\Theta}_{101} \sim \widehat{\Theta}_{104}$.

where $\operatorname{Proj}\{\cdot\}$ is the projection operator [8] and each lumped parameter is adaptively updated using the adaptation laws [17] for online estimation of unknown parameter as follows:

$$
\begin{aligned}
\operatorname{Proj}\left\{\dot{\widehat{\Theta}}_{j}\right\}= & \operatorname{Proj}\left\{\Gamma W^{T} r, \widehat{\Theta}_{j}\right\} \\
= & \begin{cases}\Gamma W^{T} r & \text { if } \hat{\Theta}_{j}>\hat{\Theta}_{-j}, \hat{\Theta}_{j}<\widehat{\Theta}_{j}, \\
\Gamma W^{T} r & \text { if } \hat{\Theta}_{j}=\hat{\Theta}_{-j}, \text { if } \Gamma W^{T} r>0, \\
\Gamma W^{T} r & \text { if } \widehat{\Theta}_{j}=\widehat{\widehat{\Theta}}_{j}, \text { if } \Gamma W^{T} r \leq 0, \\
0 & \text { elsewhere. }\end{cases}
\end{aligned}
$$

Thus, substituting $u(t)$ in (15) into $\dot{V}$ in (13) yields

$$
\begin{aligned}
\dot{V} & =r^{T} W \widetilde{\Theta}-r^{T} k_{1} r-(\dot{e}+e)^{T} e-\frac{e^{T} e}{r_{g}^{2}}+e^{T} \dot{e}-\widetilde{\Theta}^{T} \Gamma^{-1} \dot{\hat{\Theta}} \\
& =-r^{T} k_{1} r-\left(1+\frac{1}{r_{g}^{2}}\right) e^{T} e+\widetilde{\Theta}^{T}\left\{W^{T} r-\Gamma^{-1} \dot{\hat{\Theta}}\right\} .
\end{aligned}
$$

Here, $W \widetilde{\Theta}$ is defined as

$$
\begin{aligned}
W \widetilde{\Theta}= & \widetilde{M}\left(\ddot{x}_{d}+\lambda_{1} \dot{e}\right)+\widetilde{C} \dot{x}+\widetilde{D} x+\widetilde{B} \widetilde{T}_{h 1} T_{c}(\alpha) \frac{V_{J}}{V_{O}}(\alpha) \\
& +\widetilde{B} \widetilde{T}_{b 1} C_{R}(\alpha)+\widetilde{r}_{g} e+\frac{1}{2} \widetilde{M} r
\end{aligned}
$$

where $\widetilde{M}=M-\widehat{M}, \widetilde{C}=C-\hat{C}, \widetilde{D}=D-\hat{D}, \widetilde{B} \widetilde{T}_{h 1}=$ $B T_{h 1}-\widehat{B} \widehat{T}_{h 1}, \widetilde{B} \widetilde{T}_{b 1}=B T_{b 1}-\widehat{B} \widehat{T}_{b 1}, \tilde{r}_{g}=1 / r_{g}^{2}-1 / \hat{r}_{g}^{2}$, 

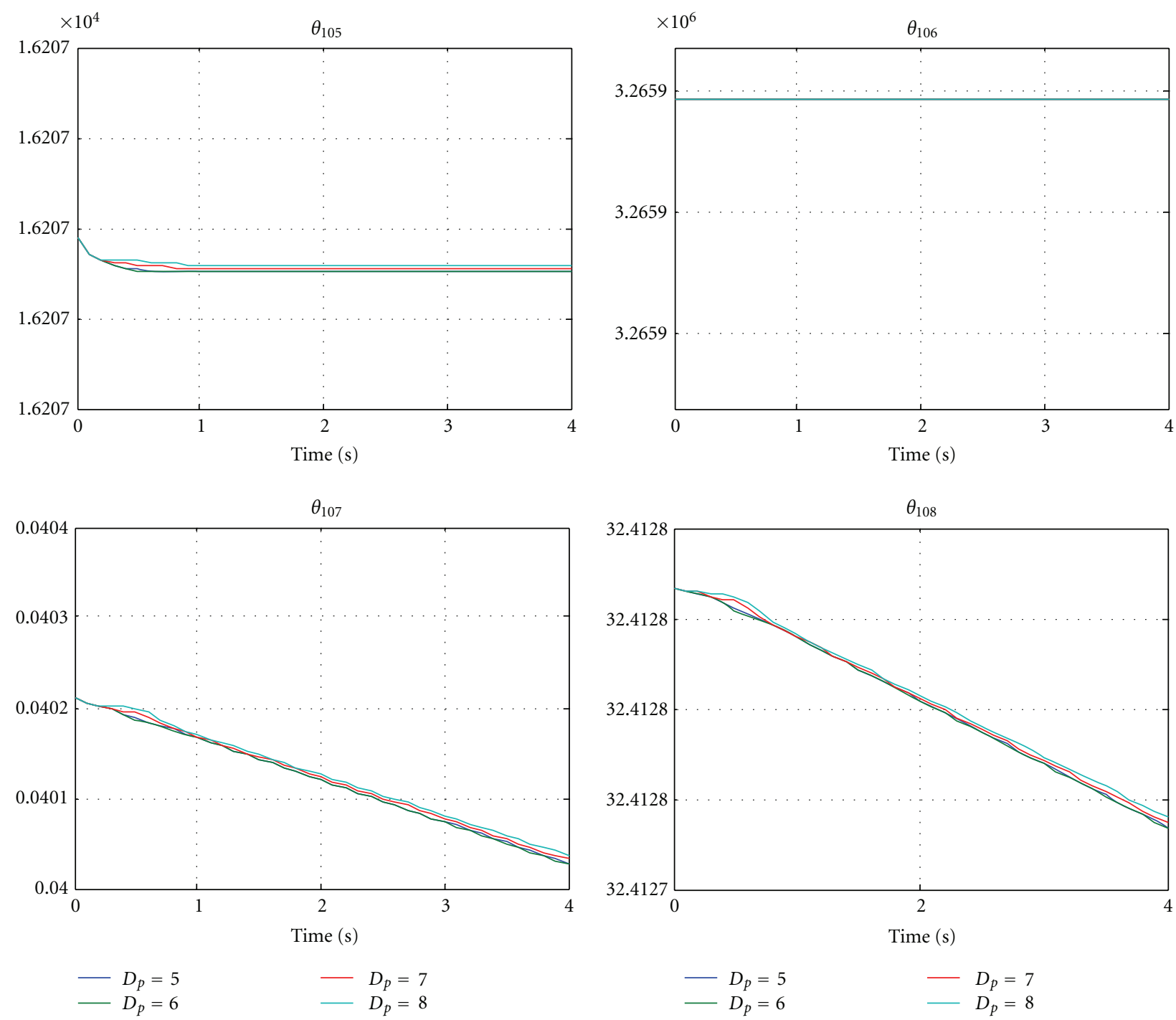

FIGURE 15: Parameter estimates: $\widehat{\Theta}_{105} \sim \widehat{\Theta}_{108}$.

and $\widetilde{M}=\dot{M}-\widehat{\dot{M}}$, in which $\widehat{T}_{h 1}=(8 / 3 \pi) \hat{\rho} \widehat{D}_{p}^{3} \widehat{V}_{O}^{2}$ and $\widehat{T}_{b 1}=$ $(\pi / 8) \hat{\mu} \widehat{D}_{s} \hat{D}_{p}^{2} \Delta \widehat{P}_{v}$. Actually, $\widetilde{\Theta}(t)$ can be expressed, for example, using the definitions of $\widehat{\Theta}$ in (16), as follows: owing to the subsequent adaptation law, the time derivative of $V(t)$ yields a negative definite function except the origin and upper bound by

$$
\dot{V} \leq-k_{1}\|r\|^{2}-\left(1+\frac{1}{r_{g}^{2}}\right)\|e\|^{2},
$$

which can be written as

$$
\dot{V} \leq-k_{2}\|z\|^{2},
$$

where $k_{2}=\min \left\{k_{1},\left(1+1 / r_{g}^{2}\right)\right\}$ is a positive constant and

$$
z=\left[r^{T}, e^{T}\right]^{T} .
$$

Using Barbalat's lemma [18], the set-point tracking error $\|z(t)\| \rightarrow 0$, thus $\|r(t)\| \rightarrow 0$ and $\|e(t)\| \rightarrow 0$ as $t \rightarrow \infty$.
Remark 1. According to the analysis from (11) to (25) of $\dot{V}$, the property of $V$, and the control law of (15) with the parameter updates of (19) and the projection-based method of update laws of (20), it is straightforward to derive a conclusion that the tracking error vector $z(t)$ in (25) is driven to zero. Thus, the set-point errors $r(t), e(t)$, and $e_{\alpha}(t)$ also vanish and the parameter estimation error vector $\widetilde{\Theta}$ in (10) is bounded where $\widehat{\Theta}$, defined after (10), is bounded due to the projection-based update method and the constant known parameter, $\Theta$. Owing to the bounds of $r(t), e(t)$ in $(8), \dot{e}(t)$ is bounded and then $x(t)$, resulting in $\alpha(t)$, and $\dot{x}(t)$ are bounded, respectively, where all desired trajectories such as $x_{d}(t)$ and $\dot{x}_{d}(t)$ are assumed to be bounded. $M(\cdot), B(\cdot), C(\cdot)$, and $D(\cdot)$ matrices in (4) are bounded because $\theta$ (due to $\Theta)$ and $x(t)$ are bounded, and $\dot{B}$ and $\dot{M}$ after (11) are also bounded owing to the bounds of $\dot{x}(t)$. $T_{\text {tot }}$ is thus bounded because $\alpha(t)$ is bounded. Hence, $W(\cdot)$ and the control input $u(t)$ are bounded and, thus, the current is bounded. This leads to the boundedness of $\ddot{x}(t)$ in the dynamic model given 

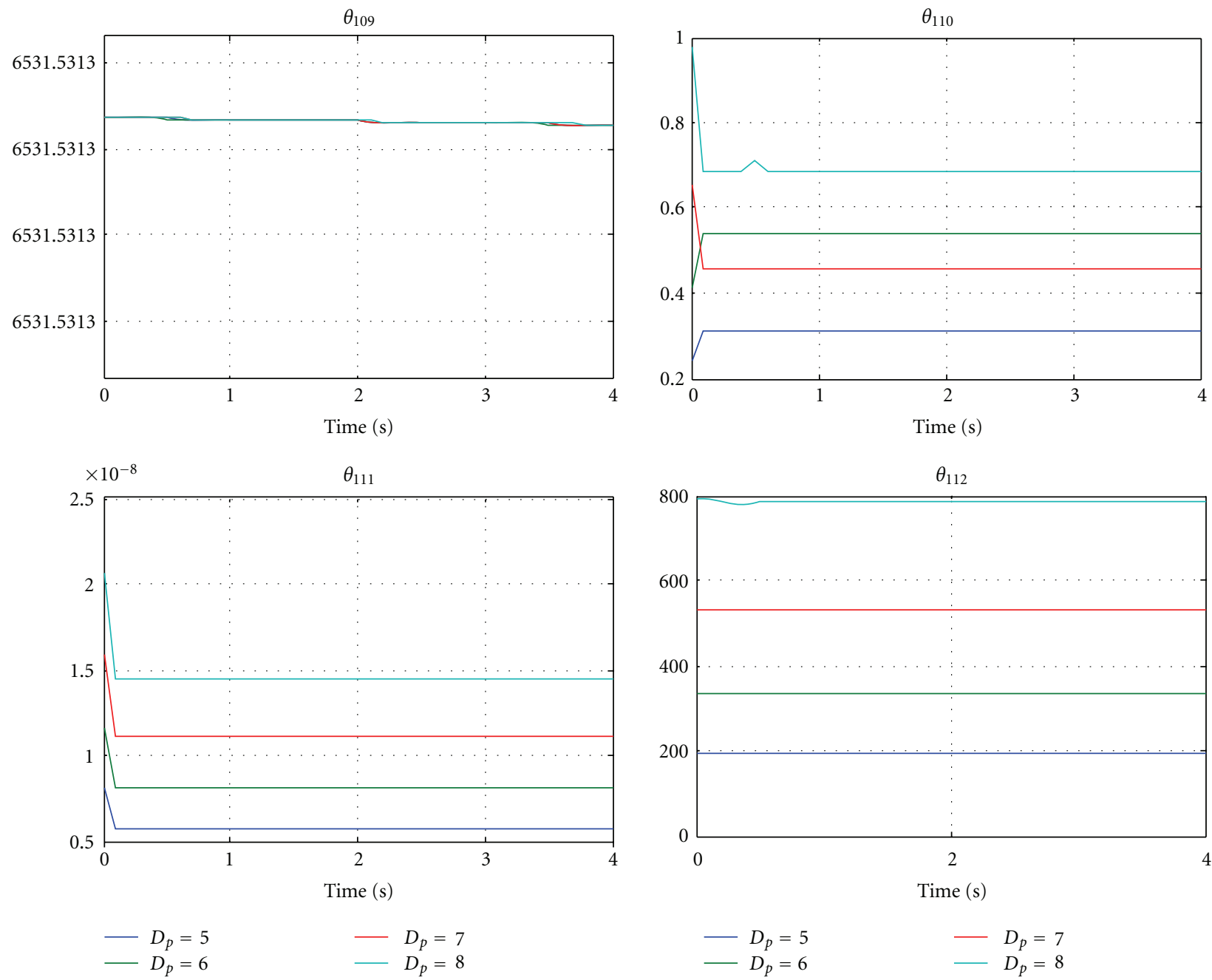

FIGURE 16: Parameter estimates: $\widehat{\Theta}_{109} \sim \widehat{\Theta}_{112}$.

in (4), which enables the boundedness of $\ddot{e}(t)$ in (6), and then the set-point tracking error dynamics $\dot{r}(t)$ in $(8)$ is bounded. Therefore, we can conclude that all signals are bounded.

Remark 2. $W \widehat{\Theta}$ in (16) where the known regression $W\left(\ddot{x}_{d}, \dot{x}\right.$, $x, r, \alpha, \dot{e}, e) \in \mathfrak{R}^{1 \times 23}$ terms and the parameter estimates $\widehat{\Theta} \in$ $\mathfrak{R}^{23}$ are given in this system as in Table 1 .

\section{Simulation Results}

Based on the dynamic model in (4), the numerical simulation is performed to verify the proposed controllers with consistently changing parameter values. The parameter values can be divided into two categories: operational values such as $D_{p}$ and $V_{O}$ and uncertain values such as $B_{1}, B_{2}$, and $\mu$. After the flow velocity $V_{O}$ is kept on $3.7[\mathrm{~m} / \mathrm{s}]$, the pipe diameter $D_{p}$ shown in Table 2 is used to vary from 5 inches to 8 inches as $D_{p}=\{5.0,6.0,7.0,8.0\}$ [in], where $D_{p}=\{0.1270,0.1524,0.1778,0.2032\}[\mathrm{m}]$, by assuming that the pipe or transmission lines are different according to their applications. With each $D_{p}$ size, the variations of all the parameters are set to $30 \%$ for the simulation results provided here. The determined parameter vectors $T_{c}(\alpha)$ and $\left[V_{J} / V_{O}\right](\alpha)$ of the butterfly valve model are borrowed from [16] for the simulation given as look-up tables from the experimental data. The amount of upper and lower variation of the unknown parameter sets is $30 \%$ of their real values. The control gains were chosen selectively as $\gamma=10, k_{1}=$ 250 , and $\lambda_{1}=1.0$ for all cases. MATLAB and Simulink are used for the simulation. The desired set-point distance of the solenoid, $x_{d}(t)$, is given as $A \cdot\left(1-e^{-B t}\right)$, where $A=0.0148$ and $B=5$.

A typical parameter set for this simulation is given by Table 2. Figures 2 and 3 show the rotation of the valve angle and the actual displacement of the plunger, respectively. This adaptive control approach shows better results compared to the results obtained from the previous research [15] using nonadaptive method, which are shown in Figures 4 and 5 for the displacement of the plunger and the angle of the butterfly valve. The developed mathematical model is the same as that of adaptive method based on the nonlinear models and the new specific approach in this paper is that the error 

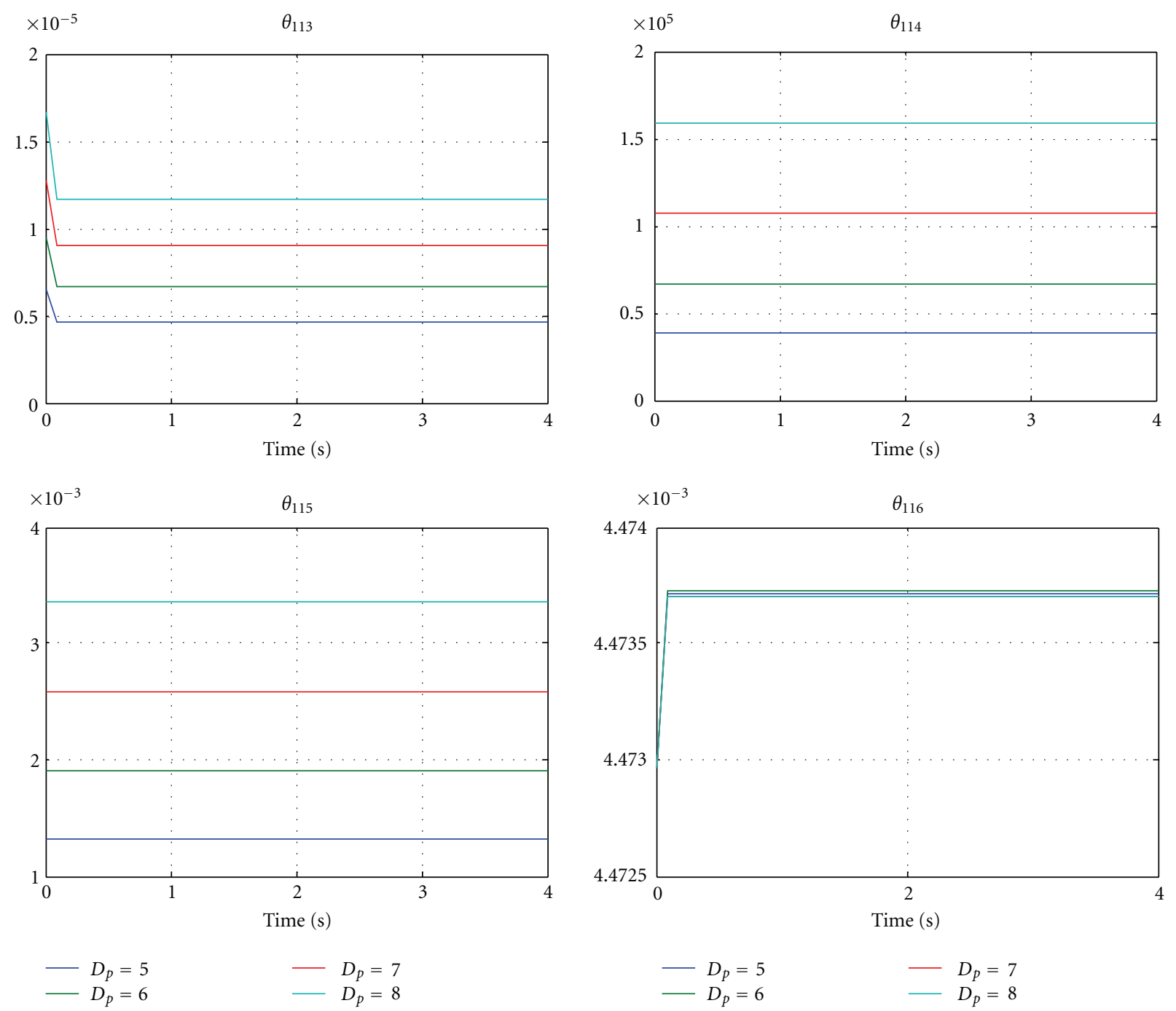

FIGURE 17: Parameter estimates: $\widehat{\Theta}_{113} \sim \widehat{\Theta}_{116}$.

signals are formulated by introducing the desired trajectory to reducing the displacement error in the adaptive method while overcoming the complicated parametric uncertainties. And also the response of the adaptive method shows that the motion process is more consistent in both variables, showing overdamped phenomena and short rising times, and then it quickly reaches the steady state. The actual movement is smoother and faster while all the parameters are varying 30\% in each flow velocity.

Figures 6 and 7 are the filtered error $r(t)$, defined in (8), and set-point error $e(t)$, given in (6), respectively. Figure 8 shows the rate of the displacement to Figure 3. From Figure 8, all figures are presented without the notation of the adaptive approach. Figure 9 shows the control input $u(t)$ of the solenoid actuator for each pipe diameter, $D_{p}$, designed in (15) with the nonlinear adaptive controller by substituting the square of the current, $i^{2}(t)$. The electromagnetic force $F_{\operatorname{mag}}(t)$ given in (2) and the total torque $T_{t}(t)$ given in (1) by summing up the hydrodynamic and bearing torques are plotted $\mathrm{i}$ n Figures 10 and 11, respectively. The torque coefficient $T_{c}(t)$ shown in Figure 12 and the ratio of input and output jet velocities shown in Figure 13 are changed for every $5^{\circ}$ of the butterfly angle. As the strokes are increased by the control input, the angles get larger and then accordingly the value of the inlet jet velocity increases, which affects the slower motion of the strokes and angles but the variables (strokes and angles) reach the steady state and the ratio as well.

The challenge is that most parameter terms in Table 1 are combined and lumped together due to the dynamic model in the presence of parameter uncertainty and the model is complicated owing to the control objectives, set-point regulation, and parametric adaptation. As given in the right side of Table 1, the unknown bounded parameter estimate vector $\widehat{\Theta} \in \mathfrak{R}^{23}$ is shown in Figures $14,15,16,17,18$, and 19 . It can be seen that the parameters such as $101,110,111,113,116$, 118 , and 121 are quickly updated in the form of premultiplication by its regression term, $W$ and go to steady state. Thus, they are more parametric-centric terms and related to the filtered error $r(t)$. The parameters such as 102, 103, 104, 105, 117,119 , and 122 are updated according to the motion of 

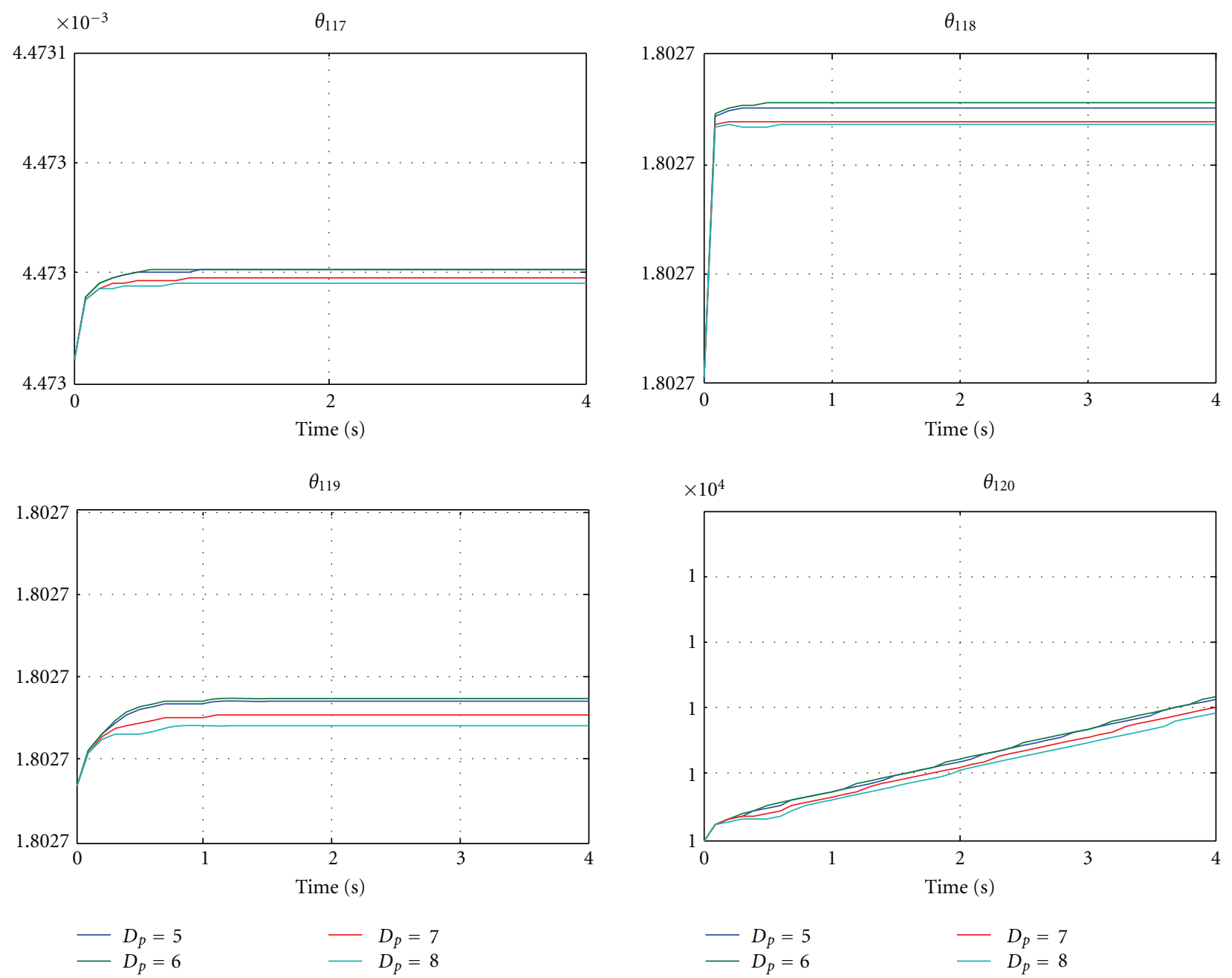

FIgURE 18: Parameter estimates: $\widehat{\Theta}_{117} \sim \widehat{\Theta}_{120}$.

the angle and stroke of the solenoid-butterfly valve system. Some parameters such as 106, 112, 114, and 115 are not updated but other parameters such as 107, 108, and 120 keep getting updated. Any parameters changing their values must be related to the control objective because these terms are incorporated into the control input in the form of the estimates.

\section{Conclusion and Future Work}

For developing advanced automation systems such as shipbased hydraulic systems, a typical solenoid-butterfly valve, which is driven by electromagnetic, fluid mechanics, and hydrodynamic forces and torques, is chosen as a continuation of previous research and an adaptive stable control approach with adaptation laws is developed accounting for uncertainties in multiple parameters on the nonlinear dynamic model. A stable adaptive controller of the solenoid-valve system is designed positioning the angle of the butterfly valve via a Lyapunov-based approach. The approach yields bounded error while adapting to the environment in the presence of complex uncertainties such as different physical appearances, uncertain parameters, operational characteristics, and parametric nonlinear dynamic models.

The parameter estimation for the unknown bounded parameters is performed using a projection algorithm whose output yields the upper and lower bounds. Numerical simulation is used to verify the performance of the proposed approach to show its effectiveness by comparing to the same dynamic model without adaptation from the previous research; when compared to the nonadaptive method, the responses of the plunger displacement and the rotating angle are steadier, smoother, and faster. Future work will be focused on demonstrating the results of hardware-in-theloop or experiments for the nonlinear solenoid valve system as well as applying the suggested adaptive method based on Lyapunov-based control approach to the real-world system. Further research on developing control techniques using robust or optimal method would be continued to overcome nonlinearities such as hysteresis or nonlinear dynamics. 

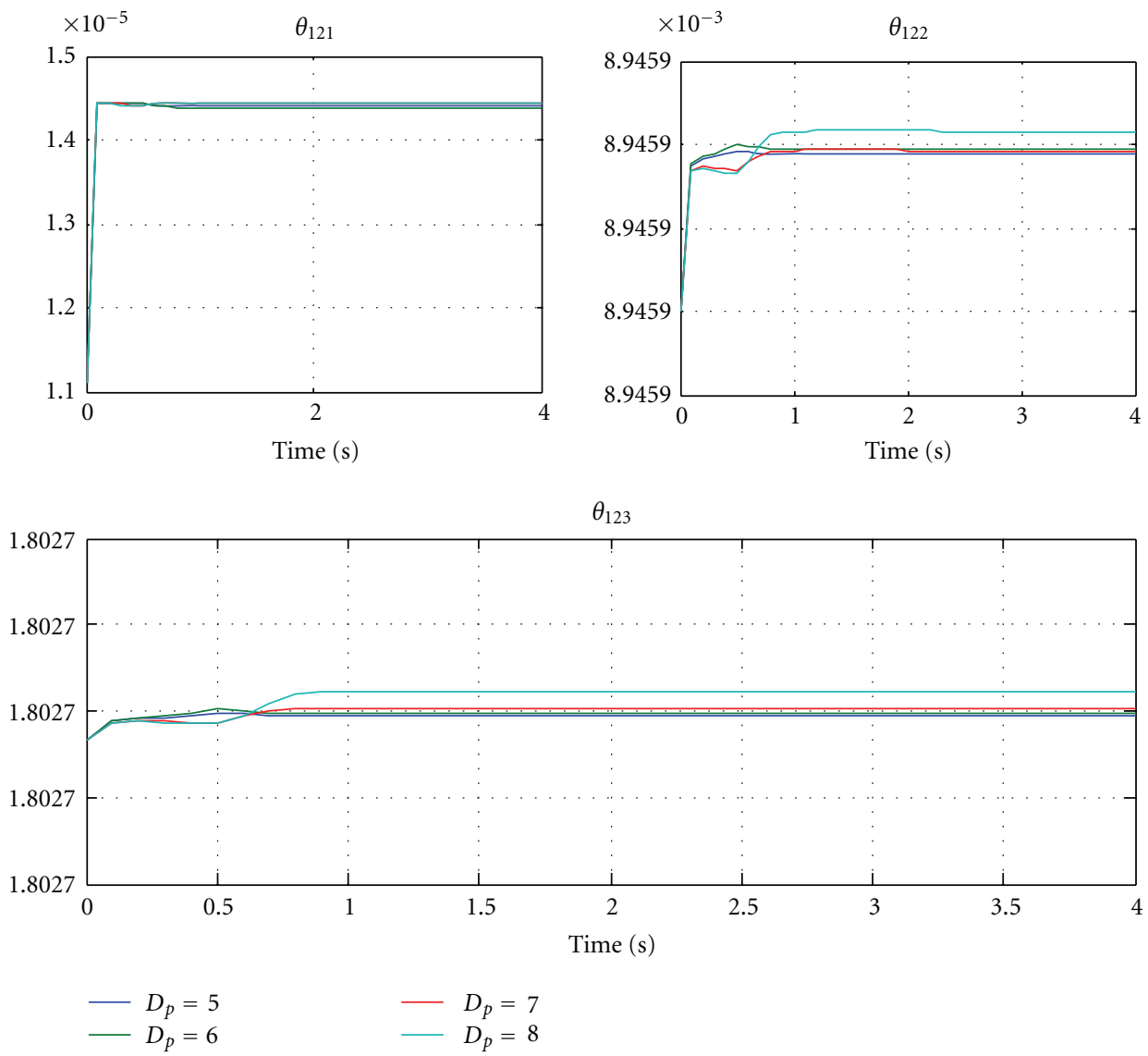

FIGURE 19: Parameter estimates: $\widehat{\Theta}_{121} \sim \widehat{\Theta}_{123}$.

\section{Nomenclature}

$B_{1}, B_{2}$ : Damping coefficients of the solenoid and butterfly valve, respectively $(\mathrm{Ns} / \mathrm{m})$

$C_{1}, C_{2}$ : Reluctances of the magnetic paths obtained from plunger geometry of solenoid actuator

$C_{R}(\alpha)$ : Bearing torque coefficient as a function of the valve angle $(\alpha)$

$D_{s}: \quad$ Stem diameter $(\mathrm{m})$

$D_{p}: \quad$ Pipe diameter (inch or $\mathrm{m}$ )

$F_{\text {mag: }}: \quad$ Magnetic force $(\mathrm{N})$

$F_{c}: \quad$ Contact force or resultant force $F_{r}(\mathrm{~N})$

$i$ : $\quad$ Current of solenoid actuator (A)

$J: \quad$ Inertia moment $\left(\mathrm{kgm}^{2}\right)$

$k$ : $\quad$ Spring stiffness $(\mathrm{N} / \mathrm{m})$

$m: \quad$ Mass of solenoid plunger $(\mathrm{kg})$

$\mu: \quad$ Friction coefficient of bearing area

$N: \quad$ Number of turns of the coil

$\Delta P_{v}: \quad$ Valve differential pressure ( $\mathrm{psi}$ )

$p: \quad$ Number of estimates

$r_{g}: \quad$ Radius of pinion gear $(\mathrm{m})$

$\rho: \quad$ Fluid mass density $\left(\mathrm{kg} / \mathrm{m}^{3}\right)$

$u$ : $\quad$ Control input $\left(\mathrm{A}^{2}\right)$
$T_{c}(\alpha):$

$T_{b}, T_{h}:$

$V_{J}, V_{O},\left(V_{J} / V_{O}\right)(\alpha)$ : Jet velocity, mean flow velocity, and their ratio as a function of the valve angle $(\alpha)$, respectively.

\section{Acknowledgments}

This research is supported by the Office of Naval Research (N00014-08-1-0435), which the authors gratefully acknowledge. Thanks are in particular due to Mr. Anthony Seman III. The authors would also like to thank Dr. Stephen Mastro and Mr. Frank Ferrese of Naval Surface Warfare Center (NSWC, Philadelphia) for help with many aspects of the paper. They are grateful to the anonymous reviewer for critical comments, which led to substantial improvement of the paper.

\section{References}

[1] A. Seman, "Adaptive automation for machinery control," in Proceedings of the Office of Naval Research (ONR) Presentation, 2007. 
[2] R. Hughes, S. Balestrini, K. Kelly, N. Weston, and D. Mavris, "Modeling of an integrated reconfigurable intelligent system (IRIS) for ship design," in Proceedings of the ASNE Ships \& Ship Systems Symposium, 2006.

[3] P. Tich, V. Mark, P. Vrba, and M. Pechoucek, "Chilled water system automation," Rockwell Automation Case Study Report, 2005.

[4] J. R. Brauer, Magnetic Actuators and Sensors, Wiley IEEE Press, Hoboken, NY, USA, 2006.

[5] Z. Leutwyier and H. Dalton, "A CFD study of the flow field, resultant force, and aerodynamic torque on a symmetric disk butterfly valve in a compressible fluid," Journal of Pressure Vessel Technology, vol. 130, Article ID 021302, pp. 1-10, 2008.

[6] T. Sarpkaya, "Oblique impact of a bounded stream on a plane lamina," Journal of the Franklin Institute, vol. 267, no. 3, pp. 229-242, 1959.

[7] T. Sarpkaya, "Torque and cavitation characteristics of butterfly valves," Journal of Applied Mechanics, vol. 29, pp. 511-518, 1961.

[8] M. Krstic, I. Kanellakopoulos, and P. Kokotovic, Nonlinear and Adaptive Control Design, Series on Adaptive and Learning Systems for Signal Processing, John Wiley \& Sons, New York, NY, USA, 1st edition, 1995.

[9] D. B. Lee, C. Nataraj, and P. Naseradinmousavi, "Nonlinear model-based adaptive control of a solenoid-valve system," in Proceedings of the ASME Dynamic Systems and Control Conference (DSCC '10), pp. 1-8, Boston, Mass, USA, September 2010.

[10] J. Pohl, M. Sethson, P. Krus, and J.-O. Palmberg, "Modelling and validation of a fast switching valve intended for combustion engine valve trains," Proceedings of the Institution of Mechanical Engineers. Part I: Journal of Systems and Control Engineering, vol. 216, no. 2, pp. 105-116, 2002.

[11] C. Nataraj, Vibration of Mechanical Systems, Cengage, 1st edition, 2011.

[12] P. Naseradinmousavi and C. Nataraj, "Nonlinear mathematical modeling of butterfly valves driven by solenoid actuators," Applied Mathematical Modelling, vol. 35, no. 5, pp. 2324-2335, 2011.

[13] M. K. Zavarehi, P. D. Lawrence, and F. Sassani, "Nonlinear modeling and validation of solenoid-controlled pilot-operated servovalves," IEEE/ASME Transactions on Mechatronics, vol. 4, no. 3, pp. 324-334, 1999.

[14] R. R. Chladny, C. R. Koch, and A. F. Lynch, "Modeling automotive gas-exchange solenoid valve actuators," IEEE Transactions on Magnetics, vol. 41, no. 3, pp. 1155-1162, 2005.

[15] C. Nataraj and P. Mousavi, "Nonlinear analysis of solenoid actuators and butterfly valve systems," in Proceedings of the 14th International Ship Control Systems Symposium, pp. 1-8, Ottawa, Canada, September 2009.

[16] J. Y. Park and M. K. Chung, "Study on hydrodynamic torque of a butterfly valve," Journal of Fluids Engineering, Transactions of the ASME, vol. 128, no. 1, pp. 190-195, 2006.

[17] J. B. Pomet and L. Praly, "Adaptive nonlinear regulation: estimation from the Lyapunov equation," IEEE Transactions on Automatic Control, vol. 37, no. 6, pp. 729-740, 1992.

[18] H. K. Khalil, Nonlinear Systems, Prentice Hall, Upper Saddle River, NJ, USA, 3rd edition, 2002. 

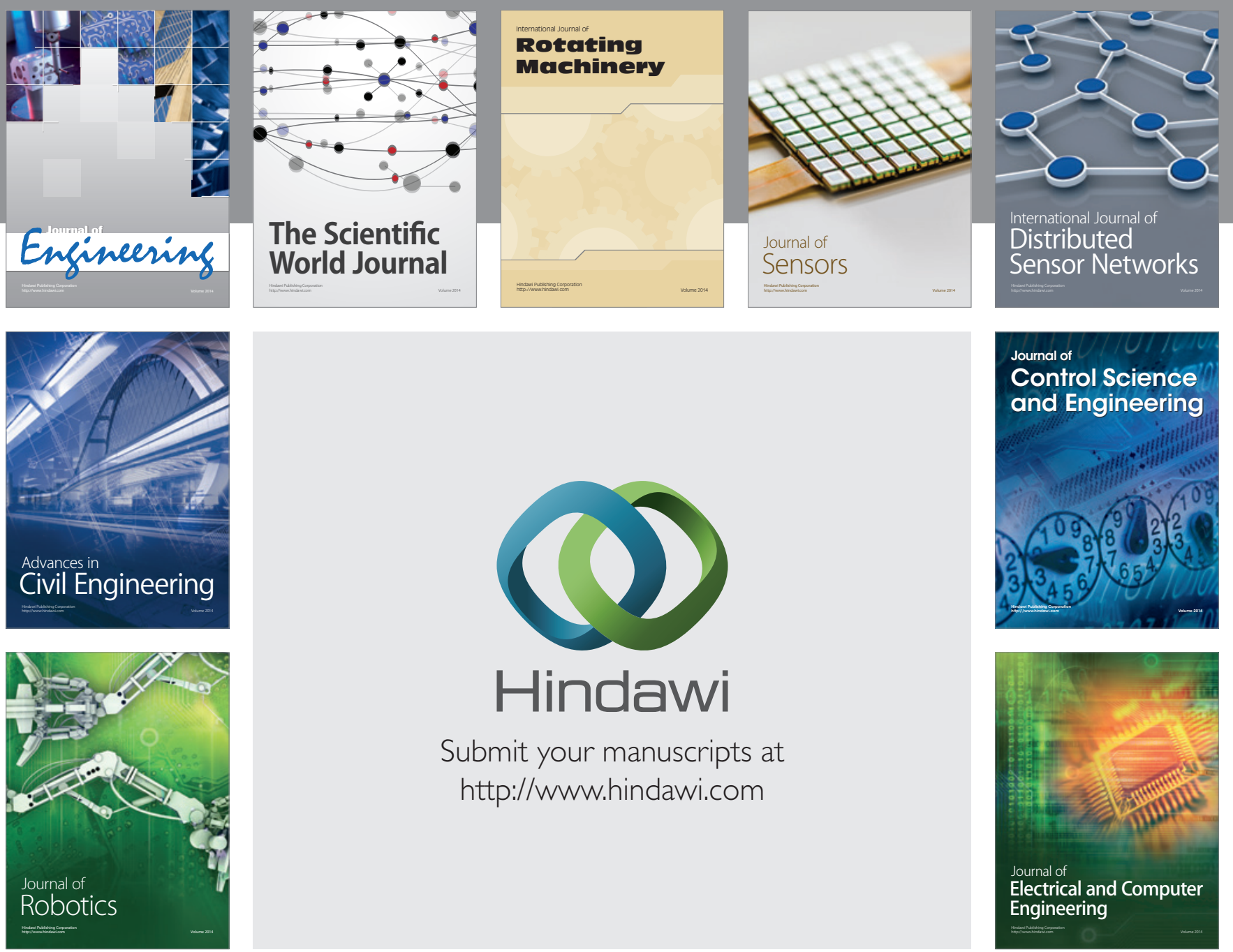

Submit your manuscripts at

http://www.hindawi.com
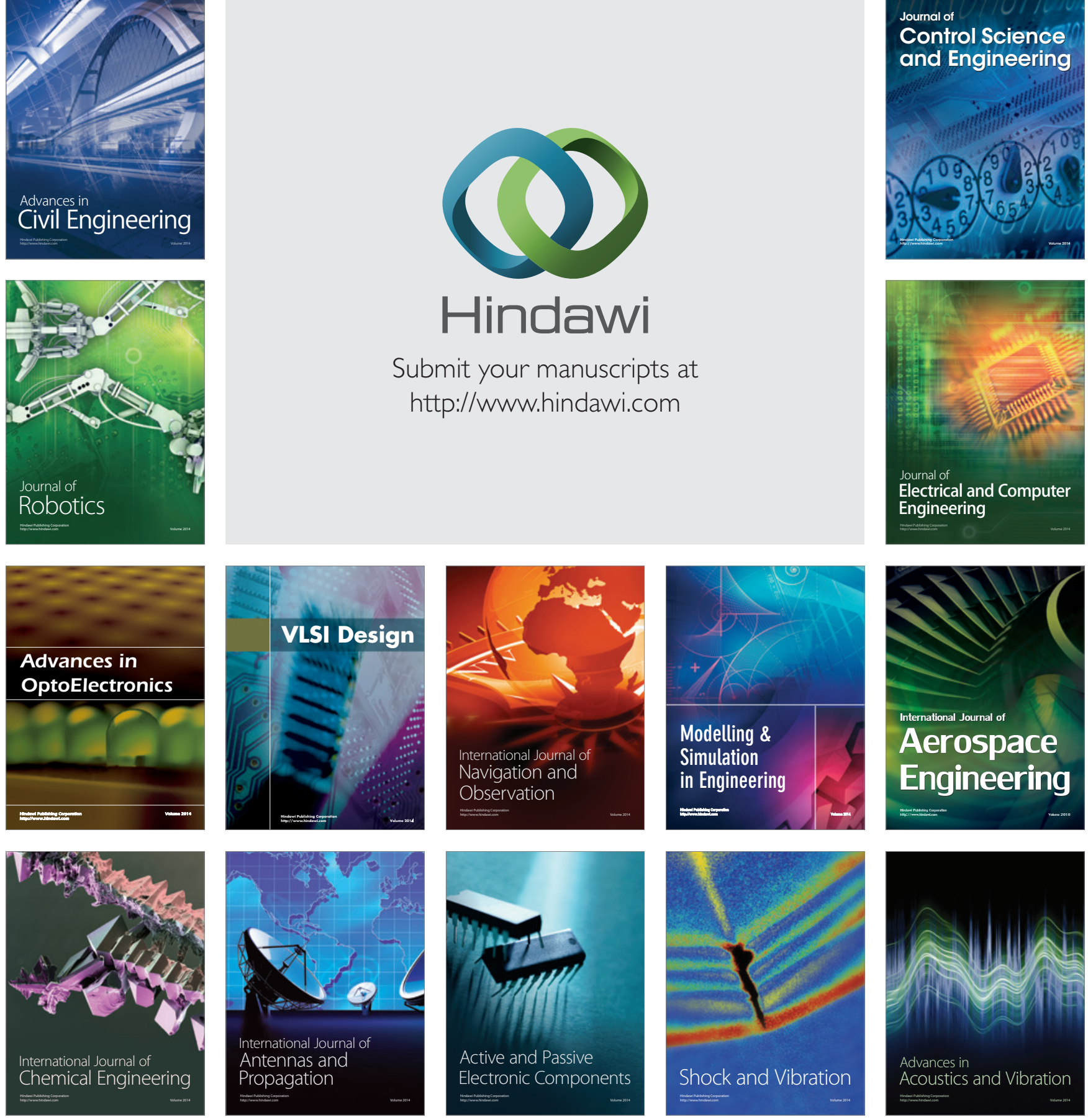